\title{
Anti-Inflammatory and Pro-Inflammatory Neuropeptides in Dilative Cardiomyopathy are there Relations to Psychophysical Stress?
}

\author{
Norbert Klein*1, Dietrich Pfeiffer ${ }^{2}$ Stefan Dhein ${ }^{3}$, Maika Klein ${ }^{2}$ and Aida Salameh ${ }^{3}$ \\ ${ }^{1}$ Department of Cardiology, Hospital St. Georg g GmbH Leipzig, Germany \\ ${ }^{2}$ Department of Cardiology, University Hospital Leipzig, Germany \\ ${ }^{3}$ Department of Cardiac surgery, University Leipzig, Heart Center, Germany
}

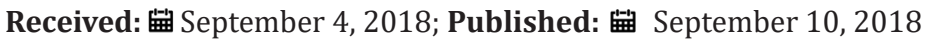

*Corresponding author: Norbert Klein, Department of Cardiology, Angiology and Internal Intensive Medicine, St. Georg Hospital Leipzig, Germany

\begin{abstract}
Background: Neuropeptides can be influenced by psychophysical stress and may have influence on cardiac function.

Objective: We wanted to find out, whether pro- and anti-inflammatory neuropeptides may be altered in heart failure (HF) and may be modulated by psychophysical stress.

Methods: Therefore, 29 patients of both gender suffering form heart failure (NYHA II-III) or and 20 healthy volunteers were investigated for the levels of the pro-inflammatory neuropeptides TNF $\alpha$, IL-6, IL-2, IL-1 $\beta$ and IL-12, and of the anti-inflammatory neuropeptides GHrelin, urocortin, adrenomedullin and VIP as well as for TH1, TH2, TH17, CD4 and CD4CD25 cells. Left ventricular (LV) ejection fraction (LVEF), end-diastolic and end-systolic diameter (LVEDD; LVESD) were assessed echocardiographically. Psychophysical stress and quality of life was evaluated by the SF-36 questionnaire.
\end{abstract}

Results: In NYHA II- and III-patients urocortin, adrenomedullin and VIP were elevated, while Ghrelin, TNF $\alpha$, IL-12 and IL-2 were unchanged. IL-6 was slightly elevated and IL- $1 \beta$ was slightly reduced in NYHA II. TH17 cells were reduced, while TH1, TH2 and CD 4 were unchanged. CD4CD25 were slightly increased. SF-36 questionnaire revealed nearly unchanged emotional and social functioning while the physical role functioning was reduced in HF-patients.

Conclusion: HF is associated with reduced quality of life as concerning physical role functioning, reduced ventricular performance and alterations of the balance between pro- and anti-inflammatory neuropeptides so that pro-inflammatory TH17 cells are reduced. Since neuropeptides also can affect cardiac output, these results indicate the activation of a neuropeptide-heart axis in HF, yet unknown. Socio-emotional stress does not seem to account for the changes.

Declaration: there is no conflict of interest of any author with any third party to declare.

Keywords: Neuropeptides; Adrenomedullin; Phenotyping Kit; Heart Failure; Hyperthyreosis; Amyloidosis; Gravidity; Implantation; Left Ventricular; Quality of Life

\section{Introduction}

Neuropeptides have gained scientific interest in the last years, in particular since they may be modulated by psychophysical stress. Among the neuropeptides two groups of peptides can be discriminated: pro-inflammatory peptides like tumour necrosis factor alpha (TNF $\alpha$ ), the interleukins IL-1 $\beta$, IL-2, IL- 6 and IL-12 and -on the other hand- anti-inflammatory peptides such as urocortin, adrenomedullin, vasoactive intestinal peptide VIP, and GHrelin. These neuropeptides and cytokines contribute to the regulation of differentiation and proliferation of immune cells like the regulatory Tcells CD4, CD4CD25, and the T-helper cells TH1, TH2 and TH17 cells. These cells participate in the adaptive immune response and regulate immune response to pathogens and on the other hand contribute to transplant rejection [1]. Another new aspect is that some neuropeptides may affect cardiac function. VIP, urocortin and adrenomedullin have been described to enhance cardiac output and to induce vasodilation [2-4].

Thus, the idea behind the present study was that a cardiac disease like dilative cardiomyopathy may have influence on the level of pro- and anti-inflammatory neuropeptides which then could alter cardiac function or the number of immunological cells such as T-helper-cells. In addition, it has been suggested that psychophysical stress may influence the neuropeptide levels [1]. Thus, one may hypothesize, that heartfailure by affecting the physical role functioning of the patient may affect his socio-emotional 
functioning, which then may affect neuropeptide expression, and thereby the immune system. Accordingly, we wanted to investigate a possible alteration of pro- and anti-inflammatory neuropeptides in heart failure (HF) and its possible modulation by psychophysical stress.

\section{Methods}

The investigation conforms with the principles outlined in the Declaration of Helsinki. The study was approved by the local ethical committee (AZ: 212-10-12072010). All patients /volunteers gave their written informed consent. The patients characteristics are given in Table 1. 29 patients of both gender (male: 17; female: 12) suffering form heart failure (NYHA II-III) or and 20 healthy volunteers (male: 12; female: 8) were enrolled in the study. Inclusion criteria were: age $>18$ and $<80$ years, heart failure NYHA II or NYHA III (patients group), absence of heart failure (control group). Exclusion criteria were: acute myocarditis, end stage heart failure, hyperthyreosis, amyloidosis, gravidity, implantation of pacemaker or ICD, immunosuppressive therapy, cancer, autoimmunologic disease, psychosis, or sepsis. These patients and volunteers were investigated for the levels of the pro-inflammatory neuropeptides TNF $\alpha$, IL-6, IL-2, IL-1 $\beta$ and IL-12, and of the antiinflammatory neuropeptides GHrelin, urocortin, adrenomedullin and VIP were analyzed by ELISA techniques using commercial ELISA kits according to the instructions of the manufacturers.

Table 1: Characteristics of the patients and volunteers. Significant differences are marked by an asterisk or hash mark ( ${ }^{*}: p<0.05$ vs. healthy; \#: p<0.05 NYHA II vs. NYHA III)

\begin{tabular}{|c|c|c|c|}
\hline & Healthy & NYHA II & NYHA III \\
\hline N & 20 & 21 & 8 \\
\hline Age (years) & $34.5 \pm 1.9$ & $58.3 \pm 2.7^{*}$ & $62.7 \pm 4.9^{*}$ \\
\hline EF (\%) & $64.7 \pm 1.3$ & $50.1 \pm 4.4^{*}$ & $39.5 \pm 6.1^{*} \#$ \\
\hline Male : Female & $12: 8$ & $13: 8$ & $4: 4$ \\
\hline $\begin{array}{c}\text { Diabetes } \\
\text { mellitus }\end{array}$ & 0 & 4 & 0 \\
\hline Hypertension & 0 & 13 & 7 \\
\hline Arrhythmia & 0 & $\begin{array}{c}1 \text { (atrial } \\
\text { fibrillation) }\end{array}$ & 0 \\
\hline
\end{tabular}

Plasma levels of neuropeptides and interleukines were measured using commercial ELISA kits: UCN, ADM and VIP (Uscn Life Science Inc, Wuhan, China), GHR (Merck Millipore, Darmstadt, Germany), IL12p40, IL2, TNFa, IL6 (eBioscience, San Diego, USA) . All samples were performed in duplicates and the concentrations were calculated relative to the standard. In order to test whether possible changes might be reflected on a cellular level, we also investigated blood lymphocytes and quantified TH1, TH2, TH17, CD4 and CD4CD25 cells using FACS analysis. Peripheral blood mononuclear cells (PBMC) were isolated from heparinized whole blood by use of Ficoll density gradient centrifugation. Th1/Th2 cells were measured by flow cytometry using commercially Th1/ Th2 Phenotyping Kit (BD, Heidelberg, Germany) and according to the manufacturer's instructions. In brief, PBMC (1x 106 cells/ ml) were stimulated in media Roswell Park Memorial Institute (RPMI) for 5 hours with phorbol myristate acetate $(50 \mathrm{ng} / \mathrm{ml})$ and ionomycin $(1 \mu \mathrm{g} / \mathrm{ml})$ in presence of protein transport inhibitor (BD,

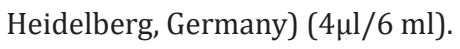

The cells were incubated with CY5.5-anti-CD4 for 20 minute and washed with PBS, then permeabilized and fixed by adding 500 $\mu \mathrm{l}$ cold Cytofix buffer. The cell pellet was re-suspended in $50 \mu \mathrm{l}$ Perm/Wash solution containing anti-IFN $\gamma$-FITC and anti-IL4-APC and incubated for $30 \mathrm{~min}$. Finally, the cells were washed with Perm/ Wash buffer and suspended in Stain Buffer until flow-cytometric analysis. All reagents were obtained from eBiosciences (San Diego, USA) and used according to the manufacturer's instructions. In brief, for cell surface staining, PBMC (1x 106 cells/ ml) were resuspended in staining buffer $(50 \mu \mathrm{l})$ and were surface stained with cocktail of anti-CD4-FITC and anti-IL2r-APC (CD25) for 30 minute at $4^{\circ} \mathrm{C}$. Then, cells were fixed by adding $100 \mu \mathrm{L}$ of fixation buffer for $20 \mathrm{~min}$ at room temperature, washed two times and incubated with anti-FOXP3 PE at room temperature for $30 \mathrm{~min}$ in the dark, followed by two washes and resuspended in $500 \mu \mathrm{l}$ until flow-cytometric analysis.

Moreover, each participant of the study was investigated echocardiographically by an experienced cardiologist. Left ventricular ejection fraction (LVEF) was determined using simpson method in apical 2- and 4-chamber-view, left ventricular end-diastolic diameter (LVEDD) and left ventricular end-systolic diameter (LVESD) were measured using M-mode in parasternal short axis. Clinical examination was performed and New York Heart Association classification (NYHA) class was evaluated. In addition, psychophysical stress and quality of life was evaluated by the SF-36 short form Quality of Life QoL questionnaire as survey tool. This survey tool comprises eight sections, which are vitality, physical functioning, bodily pain, general health perceptions, physical role functioning, emotional role functioning, social role functioning and mental health. All participants completed all eight sections. Each of the 8 scales is directly transformed into a $0-100$ scale. From this data (a) the sum score summarizing the physical role functioning parameters and (b) the sum score summarizing the social and emotional role functioning parameters was derived (according to [5]).

\section{Statistics}

All data are given as means \pm SEM of $\mathrm{n}$ individuals. For statistical analysis we performed multifactorial analysis of variance (MANOVA). If MANOVA indicated significance, a posthoc Tukey HSD test was performed. P-values $<0.05$ were considered significant. All analysis was done using Systat software (SYSTAT Version 11; Systat Inc., Evanstone, USA).

\section{Results}

Regarding echocardiographic findings HF patients as compared to healthy volunteers exhibited significantly reduced left ventricular ejection fraction (LVEF), increased endsystolic and end-diastolic diameter (LVESD, LVEDD), which correlated with the clinical degree of heart failure (NYHA II or NYHA III) $(\mathrm{p}<0.01)$ (Figure 1). The patients included suffered from dilative cardiomyopathy. ELISA revealed that among the pro-inflammatory neuropeptides the interleukins IL-2 and IL- $1 \beta$ were unchanged in HF patients 
as compared to healthy volunteers. IL-6, however, was slightly increased (Figure 2a), while IL12P40 was nearly unchanged (Figure 2b). TNF $\alpha$, which also belongs to this group of neuropeptides, was unchanged in HF patients (Figure 2b). Regarding the antiinflammatory neuropetides, it became obvious by ELISA that adrenomedullin, VIP, urocortin were all significantly increased in
HF patients in comparison to healthy volunteers (Figure 3). Only GHrelin was not altered in these patients. We next wanted to find out, whether these changes in neuropeptides might be reflected by changes on a cellular level. Therefore, we performed FACS analysis of lymphocytic cells.

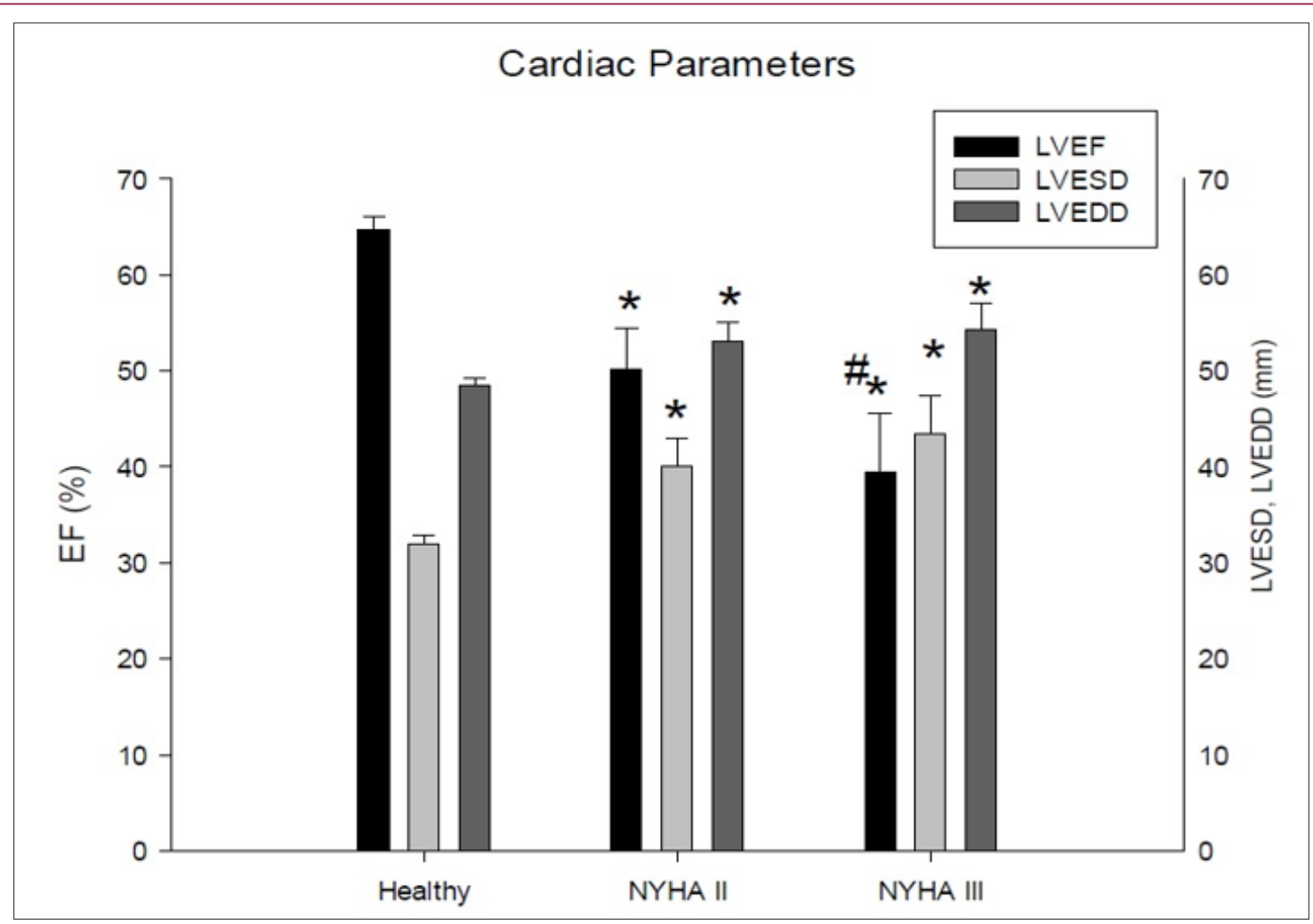

Figure 1: Echocardiographic parameters of the heart failure patients and healthy volunteers. Abbreviations: EF: ejection fraction (\%); LVEDD: left ventricular end-diastolic diameter (mm); LVESD: left ventricular end-systolic diameter (mm). Significant differences are marked by an asterisk or hash mark (*:p<0.05 vs. healthy; \#: p<0.05 NYHA II vs. NYHA III).

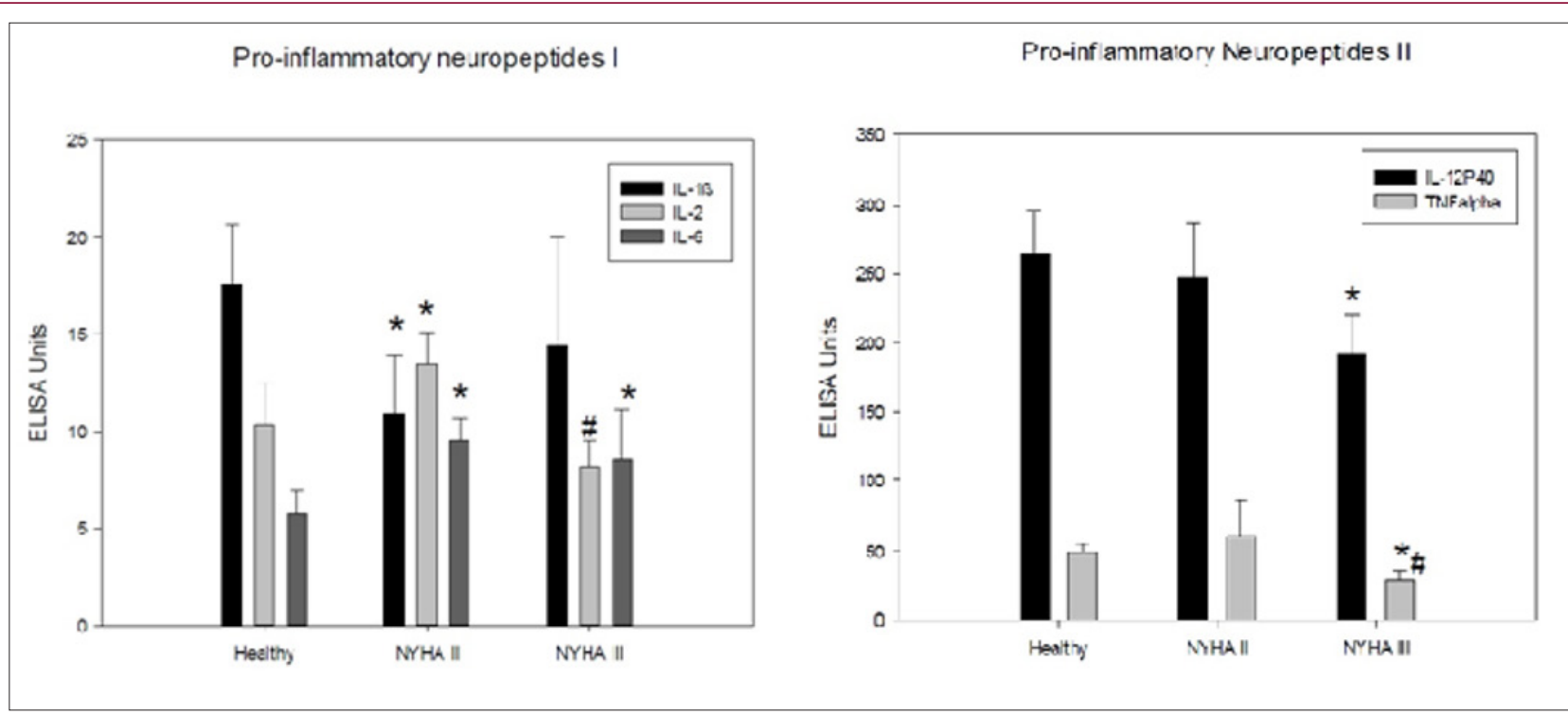

Figure 2: Panel A: Differences between healthy volunteers and heart failure patients in the blood levels as assessed by ELISA for the pro-inflammatory neuropeptides IL-6, IL-2 and IL-1 $\beta$. Panel B: Differences between healthy volunteers and heart failure patients in the blood levels as assessed by ELISA for the pro-inflammatory neuropeptides IL-12P40 and TNFa. Significant differences are marked by an asterisk or hash mark (*:p<0.05 vs. healthy; \#: $p<0.05$ NYHA II vs. NYHA III). 


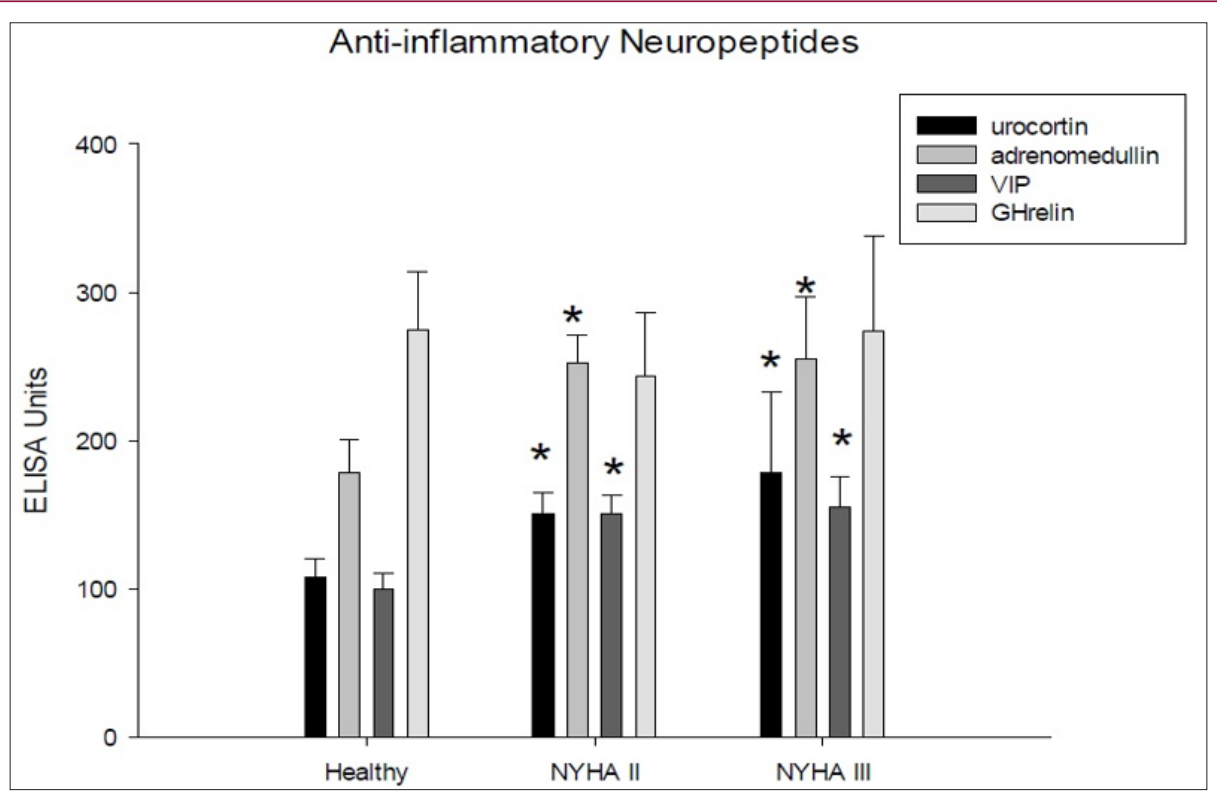

Figure 3: Differences between healthy volunteers and heart failure patients in the blood levels as assessed by ELISA for the anti-inflammatory neuropeptides VIP, Ghrelin, adrenomedullin and urocortin. Significant differences are marked by an asterisk or hash mark (*:p<0.05 vs. healthy; \#: p<0.05 NYHA II vs. NYHA III).

As outlined in Table 2, TH1, and TH2 cells were unaltered in HF patients. However, TH17 cells were significantly decreased, while CD4CD25 cells were significantly increased (Table 2). CD4+ cells were significantly increased in NYHA II, but only slightly (n.s.) in NYHA III. Since it has been hypothesized that socio-emotional stress might alter neuropeptide levels, and since one might imagine, that patients suffering from HF may exhibit a higher level of socioemotional stress, we asked all patients and volunteers to complete the SF-36 questionnaire. All patients and volunteers completed the survey. As could be expected, the sum score for physical functioning was reduced in NYHA II and even more in NYHA III patients (Figure 4). However, to our surprise these patients had no lower sum score for socio-emotional role functioning (Figure 4).

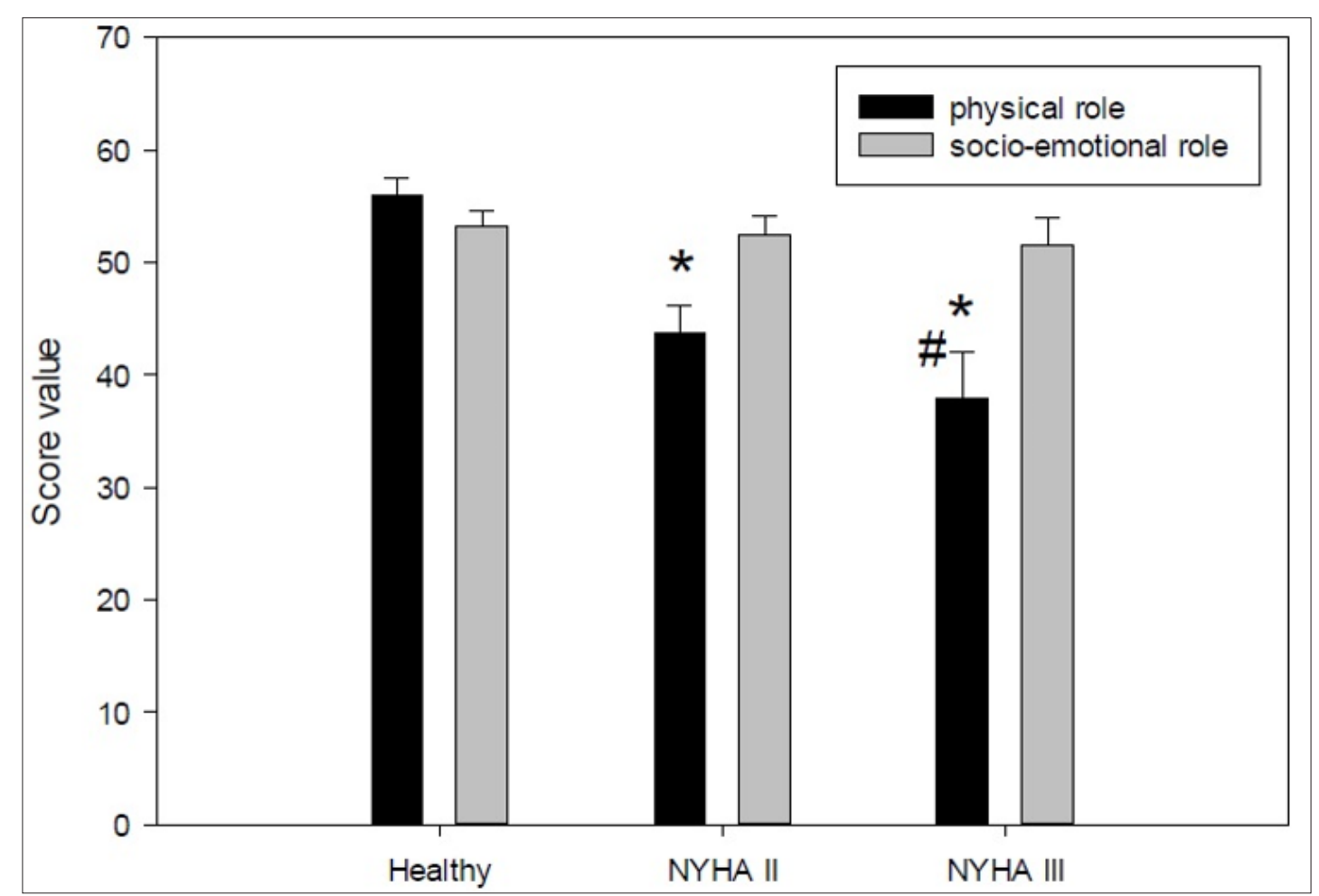

Figure 4: Quality of Life differences between healthy volunteers and heart failure patients in the blood levels as assessed by SF-36 survey. The sum score for physical role functioning and the sum score for socio-emotional role functioning are given. Significant differences are marked by an asterisk or hash mark (*:p<0.05 vs. healthy; \#: $p<0.05$ NYHA II vs. NYHA III). 
Table 2: Significant differences are marked by an asterisk or hash mark (*:p<0.05 vs. healthy; \#: p<0.05 NYHA II vs. NYHA III).

\begin{tabular}{|c|c|c|c|}
\hline & Healthy & NYHA II & NYHA III \\
\hline TH1 & $21.7 \pm 1.8$ & $25.1 \pm 2.3$ & $22.3 \pm 3.9$ \\
\hline TH2 & $1.4 \pm 0.1$ & $1.6 \pm 0.1$ & $1.7 \pm 0.6$ \\
\hline TH17 & $1.7 \pm 0.2$ & $0.7 \pm 0.1^{*}$ & $0.6 \pm 0.2^{*}$ \\
\hline CD4 & $38.6 \pm 2.7$ & $44.5 \pm 1.8^{*} \#$ & $40.2 \pm 1.6$ \\
\hline CD4CD25 & $1.1 \pm 0.1$ & $2.6 \pm 0.2^{*}$ & $2.1 \pm 0.3^{*}$ \\
\hline
\end{tabular}

\section{Discussion}

This data show, that HF is associated with a change in the balance between pro-inflammatory and anti-inflammatory neuropeptides with a shift towards the anti-inflammatory neuropeptides. Accordingly, this is associated with a reduction of pro-inflammatory TH17 cells. Interestingly, HF patients -although exhibiting lower physical role functioning score valuesdid not show reduction in the socio-emotional role (see Figure 4). This may be interpreted as an adaption of the patients to their reduced physical abilities. It needs to be taken into account that we only included NYHA II and III patients and not end-stage HF patients, i.e. NYHA IV. We had the expectation that HF patients, at least NYHA III patients, may show reduced socioemotional functioning which in turn might alter the neuropeptide system. However, the data clearly falsify this hypothesis, and the changes in the neuropeptides can not be attributed to alterations of socio-emotional role functioning. Thus, these changes must be related to other pathophysiological factors.

Thus, parallel increases in plasma adrenomedullin and in noradrenaline, atrial natriuretic peptide or interleukin- 6 have been described to be induced by prolonged exercise [6]. This is in good accordance with our data, and may be in favour of the idea that the increased activation of the sympathetic nervous system in HF and cytokine induction may contribute to the observed adrenomedullin release [7]. Adrenomedullin as a vasodilator substance is a physiological counter-regulator to sympathetic stimulation and to the renin-angiotensinaldosteron system by its vasodilator action [7,8]. Adrenomedullin can activate adenylate cyclase [9], which may evoke positive inotropic effects and may improve myocardial perfusion [10], and also may induce vasodilation [11]. Urocortin can protect the heart against ischemia/reperfusion injury [12], so that ist upregulation also could mean a counter-regulatory protective reaction in the HF patients. Release of urocortin can be induced by salt loading [13] and is released in response to stress via corticotropin releasing factor (CRF) signalling. In the cardiovascular system it can mediate increases in heart rate and vasodilation [14].

VIP, which was found to be enhanced in the HF patients in this study, typically is induced by insulin-like growth factor [15] and by sepsis [16]. VIP can increase IL-6 production by astrocytes [17] in accordance to the increase in IL- 6 found here. Moreover, VIP can induce differentiation of TH2 cells [18], reduces differentiation of TH17 to TH1 cells, but increases the proliferation of TH17 cells [19]. This, however, was not seen which may be explained by the enhancement of other neuropetides. IL- 6 can be released in response to physical exercise [20] and pathogen-associated molecular patterns (PAMPs) [21]. In the light of the literature mentioned enhanced physical exercise due to heart failure and compensatory activation of sympatho-adrenergic system may serve as one possible factor among others leading to release of IL-6 and adrenomedullin. Differentiation of dendritic cells to TH17 cells is induced by IL-6, IL-21 and IL-23 [22], while IL-4, IL-12 and IFN- $\gamma$ inhibit the proliferation of TH17.

In vitro, however, Sekiya \& Yoshimura [23] showed that IL-12 together with IFN- $\gamma$ induce TH1 cells which then can be further differentiated to TH17 cells by IL- 6 and TGF- $\beta$ (the latter only being true for mice). However, two types of TH17 cells have been discriminated: protective (Treg17) and pathogenic (Teff17) TH17 cells, and it seems that IL-6, IL-21 and TGF- $\beta$ can induce the Treg17 subtype whereas IL-6, IL-23 and IL-1 $\beta$ induce the Teff17 subtype [24]. Unfortunately we could not differentiate the TH17 cells in our study into Treg17 and Teff17 cells. The finding in our study of enhanced IL- 6 but reduced or unchanged IL- $1 \beta$ and IL-2 together with reduced IL-12 and enhanced VIP, urocortin and adrenomedullin might account for the observed reduction in TH17 cells.

A point worth discussion is the control group: we enrolled healthy volunteers, but this is associated with this group being significantly younger than the patients. However, this group has the advantage that it was free from cardiac and non-cardiac diseases. In order to circumvent the problem, that a difference might be due to the difference in age, we divided the HF group according to the NYHA stage. Only those differences between HF patients and healthy volunteers were considered relevant which also showed either similar values in NYHA II and III patients or a progression from NYHA II to NYHA III. In conclusion, HF is associated with reduced quality of life regarding the physical role functioning, reduced ventricular performance and alterations of both proand antiinflammatory neuropeptides. The balance between these neuropeptides seems to be affected so that pro-inflammatory TH17 cells, which typically are induced by pro-inflammatory neuropeptides such as IL- $1 \beta$ and inhibited by anti-inflammatory neuropeptides, are reduced.

Since pro- and anti-inflammatory neuropeptides also can affect cardiac output, these results indicate the activation of a neuropeptide-heart axis in HF, yet unknown. Overall, these changes may reflect a counter-regulatory self-protecting activation of the neuropeptides system which may limit inflammatory responses and may support cardiac function. However, the exact role of these changes in the pathophysiology of HF needs to be investigated in further in depth studies, preferably in animal HF models. Interestingly, according to our data socio-emotional stress does not seem to account for the changes in neuropeptides as the SF-36 did not reveal differences in emotional and social functioning.

\section{References}

1. Gonzalez Rey E, Delgado M (2007) Anti-inflammatory neuropeptide receptors: a new therapeutic target for immune disorders? Trends in Pharmacol Sci 28(9): 482-491.

2. Delgado M, Pozo D, Ganea D (2004) The significance of vasoactive intestinal peptide in immunomodulation. Pharmacol Rev 56(2): 249290. 
3. Brain SD, Grant AD (2004) Vascular actions of calcitonin gene-related peptide and adrenomedullin. Physiol Rev 84(3): 903-934.

4. Gravanis A, Margioris AN (2005) The corticotropin-releasing factor (CRF) family of neuropeptides in inflammation: potential therapeutic applications. Curr Med Chem 12(13): 1503-1512.

5. Ellert U, Kurth BM (2004) Methodische Betrachtungen zu den Summenscores des SF-36 anhand der erwachsenen bundesdeutschen Bevölkerung. Bundesgesundheitsbl Gesundheitsforsch Gesundheitsschutz 47: 1027-1032.

6. Tanaka M, Kitamura K, Ishizaka (1995) Plasma adrenomedullin in various diseases and exercise - induced change in adrenomedullin in healthy subjects. Internal Med 34: 728-733.

7. Krzeminski K (2016) The Role of Adrenomedullin in Cardiovascular Response to Exercise - A Review. J Hum Kinet 53: 127-142.

8. Charles CJ, Rademaker MT, Richards AM, Cooper GJ, Coy DH, et al. (2000) Adrenomedullin attenuates pressor response to angiotensin II in conscious sheep. J Cardiovasc Pharmacol 36: 526-532.

9. Sato A, Canny BJ, Autelitano DJ (1997) Adrenomedullin stimulates cAMP accumulation and inhibits atrial natriuretic peptide gene expression in cardiomyocytes. Biochem Biophys Res Commun 230: 311-314.

10. Bäumer AT, Schumann C, Cremers B (2002) Gene expression of adrenomedullin in failing myocardium: comparison to atrial natriuretic peptide. J Appl Physiol 92: 1058-1063.

11. He H, Bessho H, Fujisawa $Y$ (1995) Effects of a synthetic rat adrenomedullin on regional hemodynamics in rats. Eur J Pharmacol 273: 209-214.

12. Calderón Sánchez E, Díaz I, Ordóñez A, Smani T (2016) Urocortin-1 Mediated Cardioprotection Involves XIAP and CD40-Ligand Recovery: Role of EPAC2 and ERK1/2. PLoS One 11: e0147375.

13. Hara Y, Ueta Y, Isse T (1997) Increase of urocortin-like immunoreactivity in the rat hypothalamo-neurohypophysial system after salt loading and hypophysectomy. Neurosci Lett 227: 127-130.

ISSN: 2574-1241

DOI: 10.26717/BJSTR.2018.08.001716

Norbert Klein. Biomed J Sci \& Tech Res

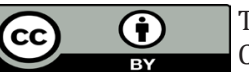

This work is licensed under Creative Commons Attribution 4.0 License

Submission Link: https://biomedres.us/submit-manuscript.php
14. Latchman DS (2002) Urocortin. The International Journal of Biochemistry \& Cell Biology 34: 907-910.

15. Lara JI, Lorenzo MJ, Cacicedo L (1994) Induction of vasoactive intestinal peptide gene expression and prolactin secretion by insulin-like growth factor I in rat pituitary cells: evidence for an autoparacrine regulatory system. Endocrinology 135(6): 2526-2532.

16. Higashiguchi T, Noguchi Y, Noffsinger A, Fischer JE, Hasselgren PO (1994) Sepsis increases production of total secreted proteins, vasoactive intestinal peptide,and peptide YY in isolated rat enterocytes. Am J Surg 168: $251-256$

17. Maimone D, Cioni C, Rosa S, Macchia G, Aloisi F, et al. (1993) Norepinephrine and vasoactive intestinal peptide induce IL-6 secretion by astrocytes: synergism with IL-1 beta and TNF alpha. J Neuroimmunol 47(1): 73-81.

18. Ganea D, Gonzalez Rey E, Delgado M (2006) A novel mechanism for immunosuppression: from neuropeptides to regulatory $\mathrm{T}$ cells. J Neuroimmune Pharmacol 1: 400-409.

19. Jimeno R, Leceta J, Martínez C (2014) Vasoactive intestinal peptide maintains the nonpathogenic profile of human th17-polarized cells. J Mol Neurosci 54(3): 512-525.

20. Pedersen BK, Fischer CP (2007) Physiological roles of muscle-derived interleukin-6 in response to exercise. Curr Opin Clin Nutr Metab Care 10(3): 265-271.

21. Tanaka T, Narazaki M, Kishimoto T (2014) IL-6 in inflammation, immunity, and disease. Cold Spring Harb Perspect Biol 6(10): a016295.

22. Jetten AM (2009) Retinoid-related orphan receptors (RORs): critical roles in development, immunity, circadian rhythm, and cellular metabolism. Nucl Recept Signal 7: e003.

23. Sekiya T, Yoshimura A (2016) In Vitro TH Differentiation Protocol. Methods Mol Biol 1344: 183-191.

24. Singh B, Schwartz JA, Sandrock C, Bellemore SM, Nikoopour E (2013) Modulation of autoimmune diseases by interleukin (IL)-17 producing regulatory T helper (Th17)cells. Indian J Med Res 138: 591-594.

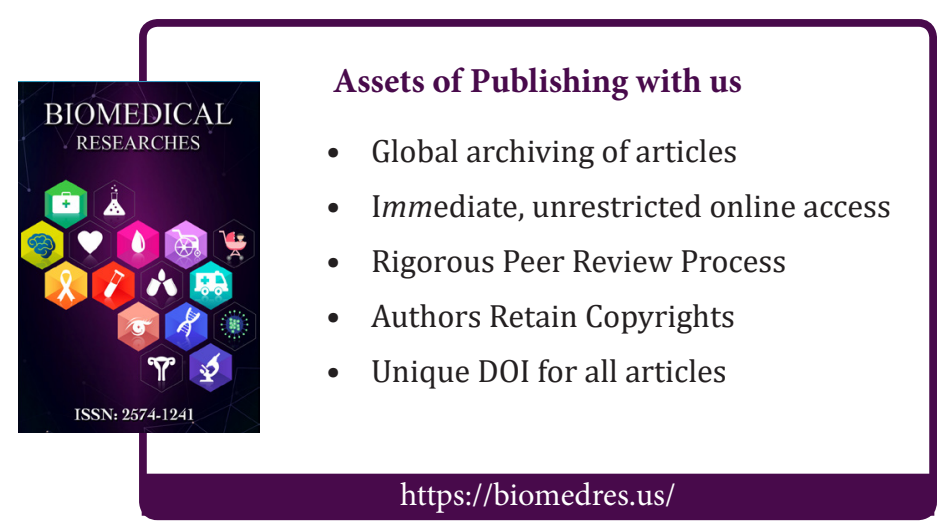

